\title{
Grid resolution control at PSDM domain tomography
}

Andrei Lache, Flor Alba Vivas and Ana Beatriz Ramirez

Copyright 2019, SBGf - Sociedade Brasileira de Geofísica

This paper was prepared for presentation during the $16^{\text {th }}$ International Congress of the Brazilian Geophysical Society held in Rio de Janeiro, Brazil, 19-22 August 2019.

Contents of this paper were reviewed by the Technical Committee of the $16^{\text {th }}$ International Congress of the Brazilian Geophysical Society and do not necessarily represent any position of the SBGf, its officers or members. Electronic reproduction or storage of any part of this paper for commercial purposes without the written consent of the Brazilian Geophysical Society is prohibited.

\section{Abstract}

PSDM domain tomography is a method that takes advantage of redundant images of the earth's subsurface. It is widely used and has become the standard modelbuilding tool for seismic depth imaging.

In this work we performed migration velocity analysis, taking advantage of a commercial software to complete part of the MVA steps required before linear inversion tomography. Ray-based tomography inversion algorithm was developed to improve inversion parameter control.

\section{Introduction}

The estimation of velocity and reflector depth from seismic data is very important when evaluating underground geologic structures to find oil and gas fields. Depth imaging requires a good velocity model to present events in their correct vertical and horizontal position; also, two additional benefits appear when Pre Stack Depth Migration (PSDM) is performed. 1) Non-hyperbolic events can be stacked and imaged. 2) Depth gathers can be used to derive an accurate velocity model (FAGIN, 2002).

Ray-based PSDM domain tomography is a widely used method that became the standard model-building tool for seismic depth imaging (WOODWARD et al., 2008). It is part of the Migration Velocity Analysis (MVA) process, which consists of iterative loops involving residual moveout (RMO) picking on PSDM gathers, tomographic ray tracing and linear updated of gridded velocity model (LAMBARE, 2014).

Different grids are involved in the process of MVA: the migration grid for offsets and space interval, the velocity grid of the initial model, the RMO grid from migrated gathers, the smoothed grid used to path ray tracing, the control point grid that include offsets interval and finally the velocity grid used in the inversion process (JONES, 2010).

The resolution of the different grids must be coherent, for example, the same range of migrated offsets must be used in the ray tracing process to obtain better match between observed and modeled depth in the inversion process.

There are different commercial software tools to perform model velocity analysis process, but in some cases, there are not enough information to understand the best grid resolution selection to each different grid, and in some cases there are no control over these parameters.
In this work, we present a technical review about the tomographic inversion process in PSDM domain, the results were obtained by a tomographic algorithm developed to control all grids parameters in the process and using synthetic data.

\section{Preparation of data to tomography inversion}

Migration velocity analysis is an iterative process that updates a starting velocity model through ray-trace grid tomography (Figure 1). The process starts with an initial velocity model (the results will be better if this initial model is near to the real model), then Pre Stack Depth Migration (PSDM) is performed in order to generate Common Image Gathers (CIGs). Next step is to carry out Residual Moveout (RMO) analysis over CIGs. When all primary reflections are aligned horizontally in all CIGs as a function of offset, the velocity model correctly focuses the seismic data, in contrast, when a wrong velocity model is used, there are observed misalignment of reflections (or residual moveouts) in the CIGs (ADLER, 2008).

Figure 1 shows the MVA cycle, where blue boxes represent the input data that is required in the MVA. Red boxes are the steps that were performed using commercial software, and black box corresponds to the tomography step that was developed with our algorithm.

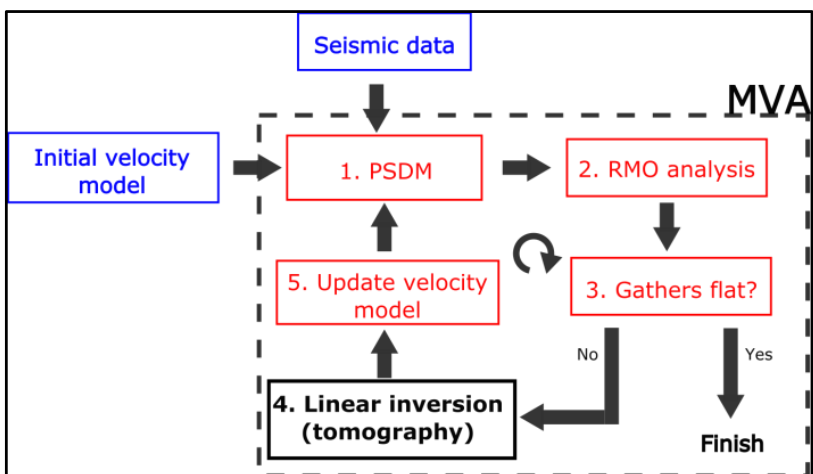

Figure 1 - Migration Velocity Analysis.

The initial velocity model for the first iteration in the MVA process is shown in the Figure 2; it was generated by processing the shot gathers of the synthetic HESS dataset. We picked velocities in the CDP domain and transform the RMS velocities to depth domain. The dimensions of the model are $72000 \mathrm{ft}$ in the offset direction and $29000 \mathrm{ft}$ of depth. 


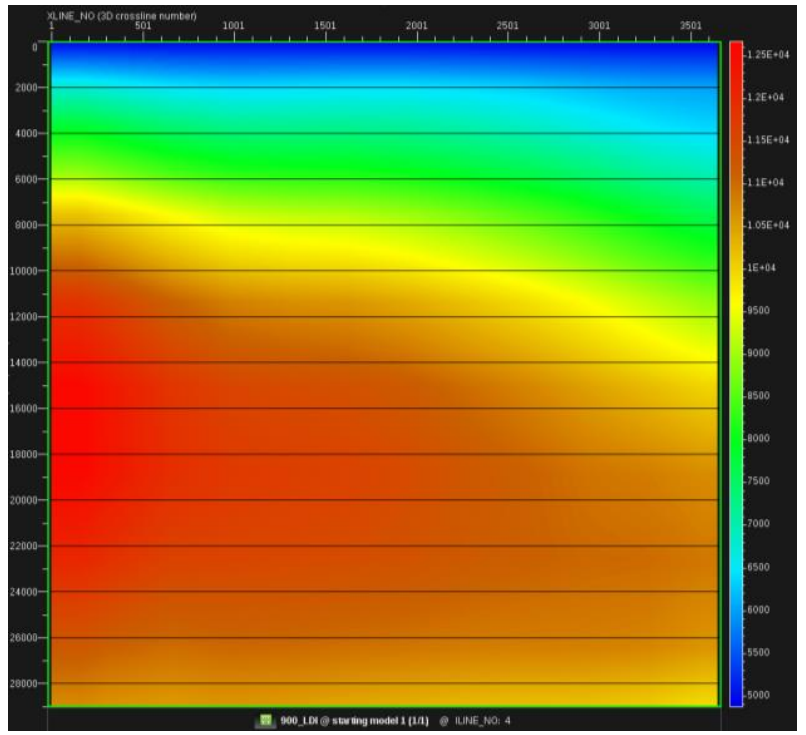

Figure 2-Starting model used in the first MVA iteration.

We started by migrating the gathers using the KDMIG module in Promax®, then we generate CIGs and obtained the RHO (Output Velocity Ratio) with the TomoMVA Auto Picker module. The $\mathrm{RHO}$ is converted to $\mathrm{RMO}$ in order to use it as the error to minimize in the tomography inversion problem.

Figure 3 shows the calculated $\mathrm{RHO}$ for the first iteration, it can be seen a strong negative error percentage at 1250 Xline and $20000 \mathrm{ft}$. In order to use these percentages in our tomography step, we converted them to residual moveout (RMO) using next equation.

$$
R M O=\sqrt{Z_{0}^{2}+R X^{2}}
$$

Where $Z_{0}$ is the reference depth or zero-offset depth, $R$ is the $\mathrm{RHO}$ percentage and $\mathrm{X}$ is the $\mathrm{Xline}$ number.

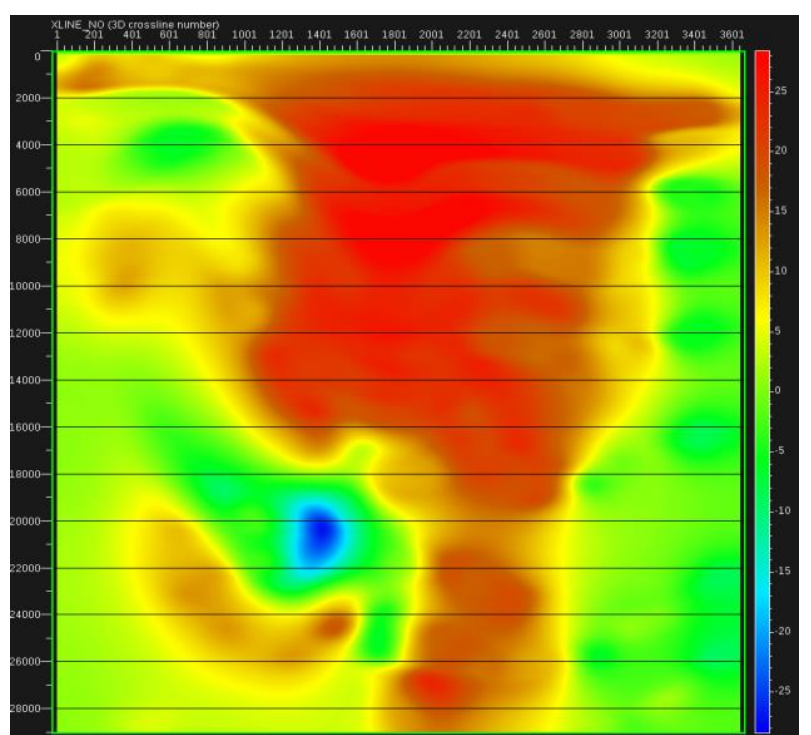

Figure 3-Calculated RHO for the inversion process.
Next step is to trace rays over the initial model (Figure 2) using TomoMVA Ray Tracing module. These rays can be generating according with control points $(\mathrm{CP})$ defined by the user. In our example we traced rays each $500 \mathrm{ft}$ in the offset and each $500 \mathrm{ft}$ in depth. Ray step length was $100 \mathrm{ft}$.

Until this part of the method, we have used commercial software to generate the information for the tomography process, so we can take advantage of these tools and focus on the construction of the inversion matrix.

\section{Tomography inversion Algorithm}

In the MVA process we define different grid resolutions in each step in the MVA iteration, the first one is the initial velocity model resolution, which usually is the finest grid and it is used in the migration step to obtain depth-seismic images, the migration usually is done with the same grid that the velocity resolution, however care must be taken in the selection of the bin center and step of the offsets. Next grid is defined by the control points spacing and it has a direct influence in the inversion equation, because it is related with the number of rows in the matrix that we need to build in the tomography step.

Another grid is selected according with the $\mathrm{RHO}$ resolution and finally the inversion cell spacing that will be the number of columns in the matrix operator for tomography. For the ray tracing step, the velocity spacing can be smoothed, if this grid is coarser, details of the velocity field will be lost.

The goal of MVA is to find the velocity model that best flattens reflections in the CIGs (SCHUSTER, 2017). RMO analysis is performed to know how much error $\left(\Delta Z=Z_{\text {event }}\right.$ - $\left.Z_{\text {ref }}\right)$ must be reduced. If the velocity model used in the MVA cycle is too fast from the real, depths away from zero offset $\left(Z_{\text {ref }}\right)$ will be too deep from the reference depth (see Figure 4), but if the velocity model used is too slow, the CIGs will show a "smile", because depths away from zero offset will be too shallow from reference. These errors are the input for the tomographic inversion problem that the method will try to resolve.

According with Robein (ROBEIN, 2010) the gradients $\delta \Delta Z / \Delta m_{i}$ tell us how much a given residual moveout $\Delta Z$ somewhere in the gathers changes if one perturbs one single parameter $\mathrm{m}_{\mathrm{i}}$ of a small quantity $\mathrm{dm}_{\mathrm{i}}$ somewhere in the initial model.

$$
\left[\frac{\partial \Delta Z_{j}}{\partial m_{i}}\right]_{M \times N} \cdot\left[d m_{i}\right]_{N \times 1}=-\left[\Delta Z_{i}\right]_{M \times 1},
$$




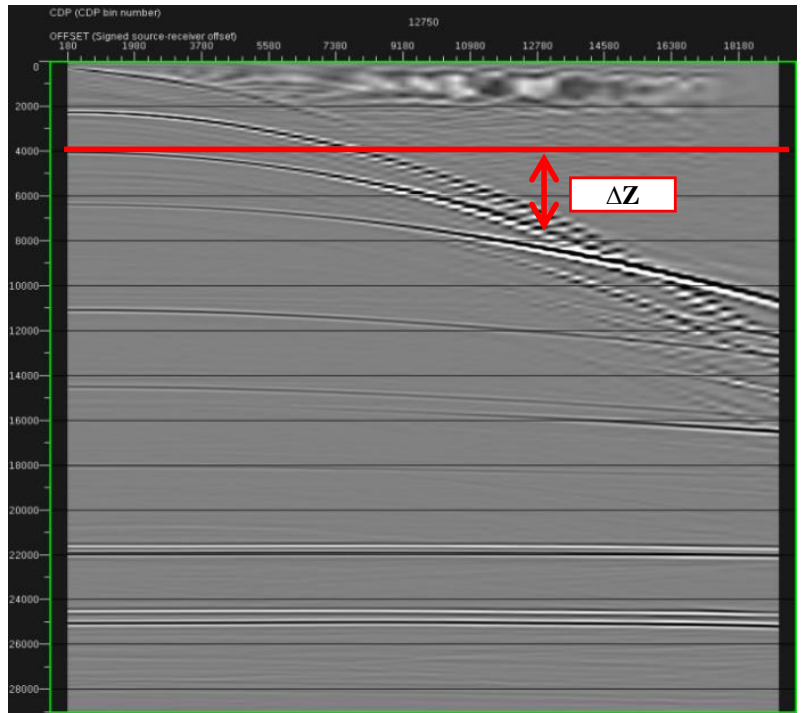

Figure 4-Depth gather pattern when initial velocity model is fastest than true velocity model.

Our Objective was to build the gradients matrix and perform the inversion process using the conjugate gradients iterative solver. We started defining the gradients values according with STORK (1992), XIA et al (2008) and MENG et al (2004).

$$
\frac{\partial \Delta Z}{\partial t}=\frac{1}{2 s \cos \alpha \cos \gamma},
$$

and,

$$
\frac{\partial t}{\partial m}=\left[L_{h}-\left(L_{h=0} \cos \gamma\right)\right],
$$

Where $s$ is the local slowness above the reflector at the point of reflection, $\alpha$ is the local dip of the reflector, $\gamma$ is the angle of incidence of the ray on the reflector measured from the reflector's perpendicular, $L_{h}$ is the path length of a specularly reflected ray having offset ray parameter $h$ at a given reflection point and $L_{n=0}$ is the path length when the offset is zero.

Conjugate gradient algorithm was implemented to resolve the inverse problem. The conjugate gradient method is easily adapted to take advantage of the sparsity of the problem (SCALES, 1987).

\section{Tomography velocity update with synthetic data}

Ray tracing and RHO information was available in an ASCII file. Our algorithm reads each ray and takes into account its incidence angle and its total length for the matrix construction, also a grid resolution is defined and the $\Delta Z$ vector is built for the inversion process.

Figure 5 shows delta velocity calculated with the first MVA iteration. It can be seen that tomography tries to find the best velocity perturbation to flatten the gathers. Conjugate gradient method needed 20 iterations to obtain a residual of $4 \times 10-3$. In Figure 6 is presented the first updated velocity model which is the result of add the initial velocity model
(Figure 2) and the delta model calculated after the first MVA iteration (Figure 5).

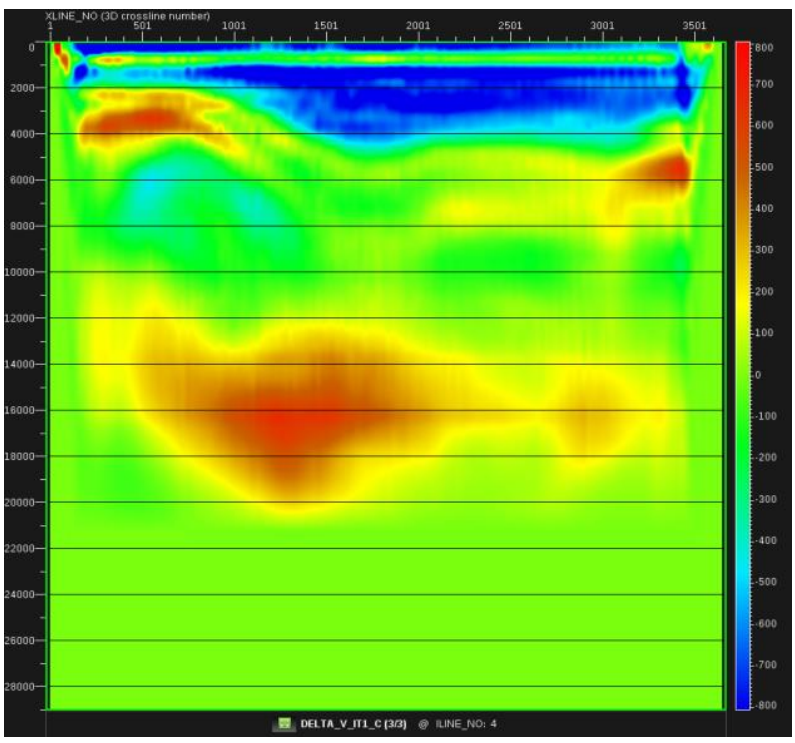

Figure 5 -Delta velocity for the first MVA iteration.

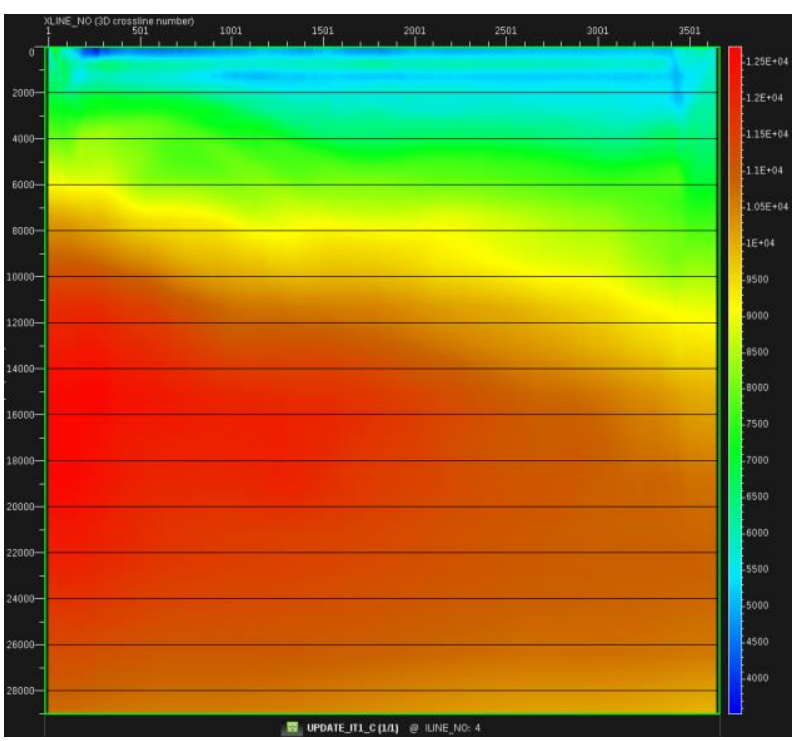

Figure 6 - Updated velocity model first iteration.

Figures 7 and 8 show the improvement performed by the first MVA iteration for two different ClGs. In Figure 7 the initial velocity model was used to perform migration and in Figure 8 the migration was done with the updated velocity model, it can be seen that events are flatten in comparison with Figure 7. 


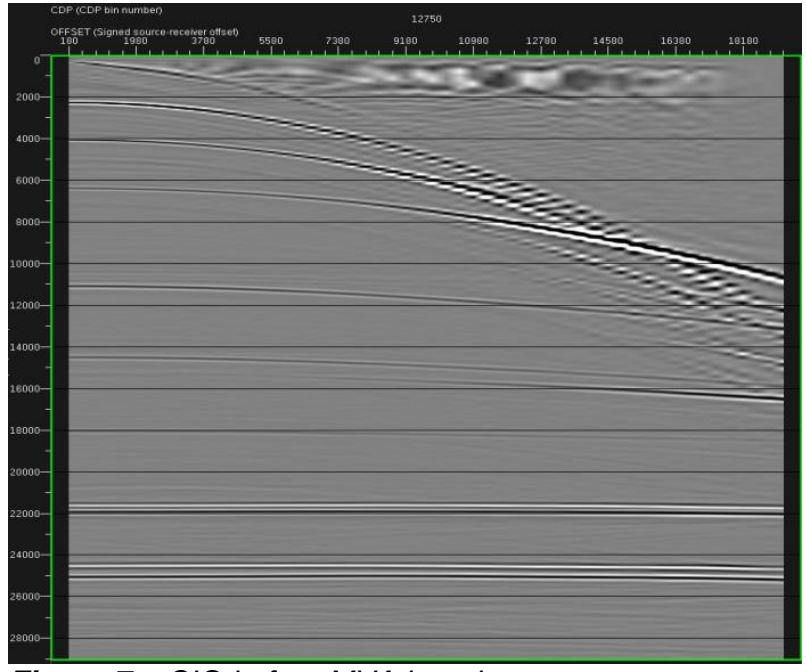

Figure 7-CIG before MVA iteration.

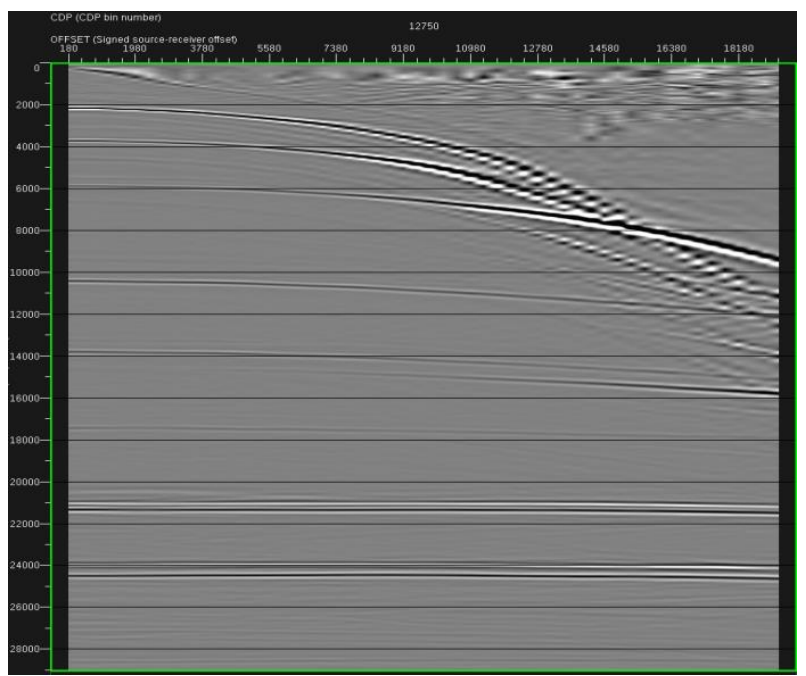

Figure 8-CIG after first MVA iteration.

Next Figure shows the updated velocity model obtained after three MVA iterations.

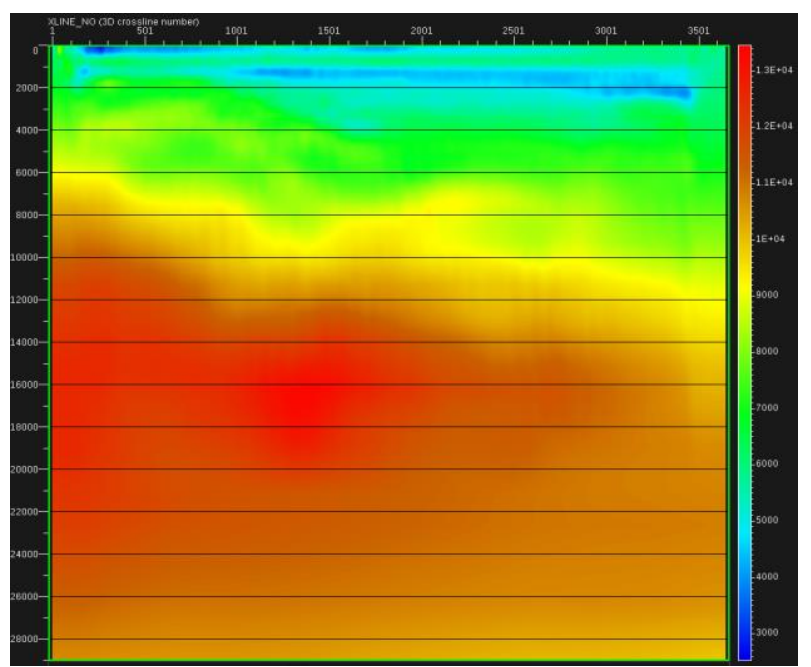

Figure 9 - Updated velocity model after three MVA iterations.

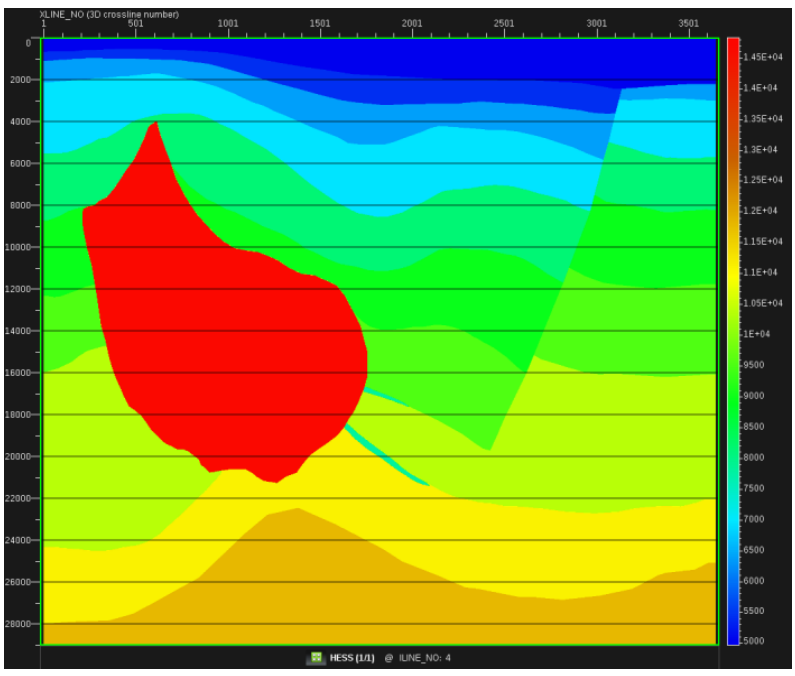

Figure 10 - HESS velocity model.

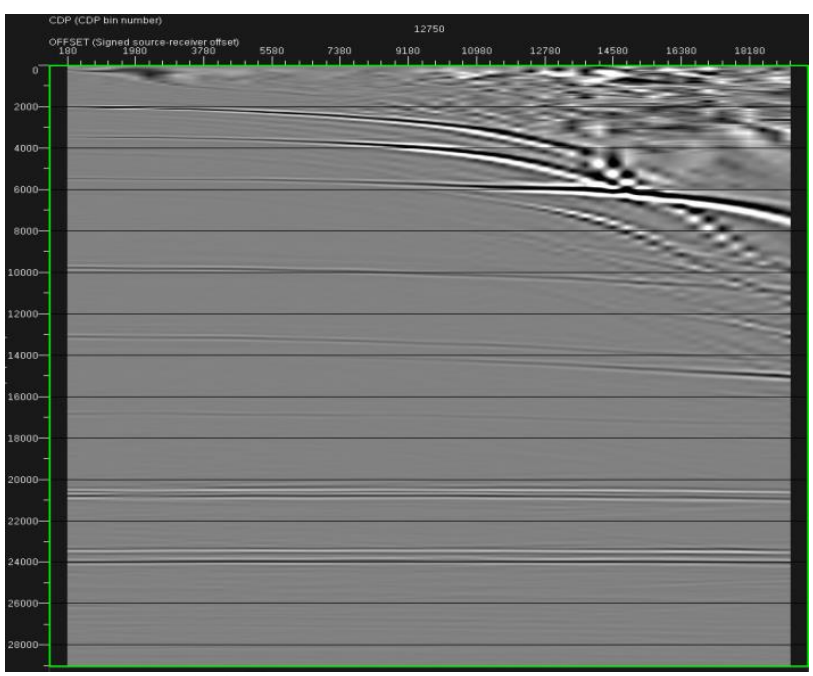

Figure 11 - CIG after three MVA iterations.

Figure 11 was obtained using the velocity model after three MVA iterations. It is observed that events are flatten for the same CIG presented in Figure 7 and 8.

\section{Conclusions}

Control points spacing and inversion grid resolution define matrix dimensions in the tomography problem.

More than one MVA iterations are often required in order to flatten correctly events in CIGs.

We have applied PSDM tomography successfully to a synthetic dataset, the method will be suitable for velocitydepth-complex models for very large seismic datasets.

\section{Acknowledgments}

This work is supported by the Colombian Oil Company ECOPETROL and CPS research group of Industrial University of Santander. 


\section{References}

ADLER, F., BAINA, R., SOUDANI, M., CARDON, P. and RICHARD, J. Nonlinear 3D tomographic least-squares inversion of residual moveout in Kirchhorff prestack-depthmigration common-image gathers. Geophysics, vol 73, no. 5, pp. VE13-VE23, 2008.

FAGIN, S. Model-Based Depth Imaging, $2^{\text {nd }}$ ed. Society of Exploration Geophysicists, 2002.

JONES, I. Tutorial: Velocity estimation via ray-based tomography. First Break. Vol 28, pp. 45-52. 2010.

LAMBARE, G., GUILLAUME, P., MONTEL, J.P. Recent advances in ray-based tomography. EAGE conference and exhibition, 2014.

MENG, Z., VALASEK, P., WHITNEY, S., SIGLER, C., MACY, B. and WHITMORE, N. 3D Global tomographic velocity model building. 74th Annual International Meeting, SEG, Expanded Abstracts, 2379 - 2382. 2004.

ROBEIN, E. Seismic Imaging. A Review of the Techniques, their Principles, Merits and Limitations. EAGE. 2010.

SCALES, J. Tomographic inversion via the conjugate gradient method. Geophysics, vol 52, no. 2, pp. 179-185. 1987.

SCHUSTER, G. Seismic Inversion. Society of Exploration Geophysicists. 2017.

STORK, C. Reflection tomography in the postmigrated domain. Geophysics, vol 57, no. 5, pp. 680-692, 1992.

WOODWARD, M.J., NICHOLS, D., ZDRAVEVA, O., WHITFIELD, P. and JOHNS, T. A decade of tomography. Geophysics, vol 73, no. 5, pp. VE5 - VE11. 2008.

XIA, FAN., REN, Y. and JIN, S. Tomographic migrationvelocity analysis using common angle image gathers. SEG Annual Conference, Las Vegas, Nevada, (Nov. 9, 2008), pp. 3103-3107. 